Artículo

Los textos publicados son responsabilidad exclusiva de sus autores

\title{
Atención plena disposicional y estilos de manejo de conflictos en estudiantes universitarios
}

\section{Dispositional mindfulness and conflict handling styles in undergraduate students}

\author{
Alejandro César Antonio Luna Bernal \\ Departamento de Filosofía \\ Universidad de Guadalajara \\ Doctor en Psicología \\ https://orcid.org/0000-0002-3371-5999 \\ alejandro.luna@academicos.udg.mx \\ Josefina Sandoval Martínez
Universidad de Guadalajara
josefina.sandoval@academicos.udg.mx
https://orcid.org/0000-0002-4113-1897 \\ Alejandra De Gante Casas \\ Universidad de Guadalajara \\ alejandra.degante@academicos.udg.mx \\ https://orcid.org/0000-0001-7186-1359
}

\section{Referencia}

Luna Bernal, A. C. A., Sandoval Martínez, J. y De Gante Casas, A. (2021). Atención plena disposicional y estilos de manejo de conflictos en estudiantes universitarios. Revista Guatemalteca de Educación Superior, 4(2), 50-60. DOI: https://doi.org/10.46954/revistages.v4i2.62

Recibido: 15/02/2021

Aceptado: 04/04/2021

\section{Resumen}

El objetivo del estudio fue analizar la relación entre la atención plena disposicional y los estilos de manejo de conflictos (dominante, complaciente y cooperativo) usados por jóvenes universitarios de pregrado, en las relaciones con sus compañeros de escuela. Participaron 301 estudiantes de una universidad pública del occidente de México, con edades de 17 a 25 años, quienes respondieron el Inventario de Friburgo sobre Atención 
Plena, versión corta y el Cuestionario sobre Estilos de Mensajes en el Manejo de Conflictos. Los resultados mostraron niveles moderados de atención plena disposicional y estilo cooperativo entre los participantes. La atención plena disposicional presentó una correlación positiva directa estadísticamente significativa, de moderada a fuerte, con el estilo cooperativo, así como una correlación significativa inversa, aunque débil, con el estilo dominante. Se discuten estos resultados destacando su congruencia con estudios previos. Se concluye señalando el valor del presente estudio como una aportación de interés para el diseño de futuros programas de investigación e intervención que pudieran desarrollarse con jóvenes, especialmente en el contexto universitario.

\section{Abstract}

The aim of this study was to analyze the relationship between dispositional mindfulness and conflict management styles (dominant, complacent, and cooperative) used by undergraduate college students in their relationships with their schoolmates. The participants were 301 students from a public university in western Mexico, aged 17 to 25 years. They answered the Ross-DeWine Conflict Management Message Style Instrument and the Freiburg Mindfulness Inventory-short version. The findings showed moderate levels of dispositional mindfulness and cooperative style among the participants. Dispositional mindfulness presented a statistically significant direct positive correlation, from moderate to strong, with the cooperative style, as well as a significant inverse correlation, although weak, with the dominant style. These results are discussed, highlighting their consistent with previous studies. It concludes by pointing out the value of this study as an interesting contribution to the design of future research and intervention programs that could be developed with young people, especially in the university context.

\section{Introducción}

De acuerdo con Toniolo-Barrios et al. (2020), las investigaciones sobre atención plena (mindfuness) constituyen actualmente, a nivel internacional, un campo extraordinariamente prolífico

\section{Palabras clave:}

atención plena disposicional, estilos de manejo de conflictos, estudiantes universitarios.

\section{Keywords:}

conflict handling styles, dispositional mindfulness, undergraduate students. 
el cual "crece constantemente como una fuente confiable de investigación científica académica y rigurosa" (p. 1077). Una de las razones que explican este interés en áreas como la medicina, la psicología y la educación se debe a los efectos positivos que esta capacidad ha mostrado tener, de manera consistente, en la promoción de estados de salud y bienestar individual y social, tal como se ha documentado en trabajos recientes de revisión sistemática de la literatura académica (por ejemplo, Soriano et al., 2020; López-Hernáez, 2016). De ahí el interés de la presente investigación por explorar su posible papel en los procesos de gestión constructiva de los conflictos en el espacio escolar universitario.

De acuerdo con Levesque (2018), la atención plena hace referencia fundamentalmente a "la conciencia que emerge de prestar atención; esa conciencia se desarrolla al prestar atención a propósito, enfocarse en el momento presente y hacerlo sin juzgar a medida que se desarrolla la experiencia" (p. 2349). No obstante, el término mindfuness presenta cierta ambigüedad, ya que puede entenderse como una capacidad que se desarrolla de manera espontánea en las personas (atención plena disposicional o mindfulness disposicional) y, por otro lado, también se usa para hacer referencia a una práctica de meditación que busca de manera deliberada fortalecer e incrementar dicha capacidad (meditación mindfulness) (Laca et al., 2017). La presente investigación se centra en el primer sentido mencionado, aunque es importante no perder de vista la relación entre ambos debido a que "el mindfulness puede encuadrarse como un rasgo disposicional del individuo, que puede perfeccionarse por medio de protocolos de entrenamiento" (Ramírez-Garduño et al., 2020, p. 4).

Por otra parte, los estilos de manejo de conflictos pueden definirse como "las maneras en que las personas se comportan habitualmente frente a situaciones de conflicto" (Luna y Laca, 2014 , p. 38). Para investigar esta variable, en el presente estudio se tomó como base el modelo propuesto por Ross y De Wine (1988), de acuerdo con el cual es posible definir tres tipos de mensajes verbales con que los jóvenes gestionan sus discusiones con otras personas: a) el estilo enfocado en símismo o dominante, 
se produce cuando el individuo usa mensajes centrados en sus propias demandas, con una actitud impositiva frente a los demás; b) el estilo enfocado en la otra parte o complaciente, donde los mensajes verbales se centran en el bienestar de la contraparte y se orientan a satisfacer sus necesidades; y, c) el estilo enfocado en el problema o cooperativo, en el que el joven invita a la otra persona a realizar un esfuerzo conjunto para solucionar la cuestión en conflicto. Este modelo de Ross y De Wine (1988) ha sido ya anteriormente utilizado en estudios con jóvenes hispanohablantes (Mejía y Laca, 2006; Laca y Mejía, 2017; Luna y Laca, 2014).

En congruencia con lo señado, se establecieron los siguientes dos objetivos específicos para la presente investigación. En primer lugar, calcular las puntuaciones de los participantes en cada una de las variables de estudio (atención plena disposicional y estilos de manejo de conflictos), a fin de conocer el grado en el que éstas se presentan entre los jóvenes universitarios. En segundo lugar, analizar las relaciones entre los estilos de manejo de conflictos, y la atención plena disposicional.

\section{Materiales y métodos}

Participaron 301 estudiantes de una Universidad pública del occidente de México, matriculados en tres programas de Licenciatura (Psicología, Medicina, y Trabajo Social), y uno de Técnico Superior Universitario (TSU en Prótesis Dental). El muestreo fue no probabilístico e intencional. El rango de edad fue de 17 a 25 años, con media aritmética de 20.58 y desviación estándar de 1.84.

Los participantes respondieron dos instrumentos psicométricos. El primero fue el Inventario de Friburgo sobre Atención Plena, versión corta (Freiburg Mindfulness Inventory, FMI-14; Walach et al., 2006). Se utilizó la versión traducida y validada al español por Pérez-Verduzco y Laca-Arocena (2017). Es un cuestionario de 14 reactivos con una escala Likert de cuatro puntos desde $1=$ Raramente hasta 4 = Casi siempre. La puntuación se obtiene calculando la media aritmética de las respuestas a los 14 
reactivos. La confiabilidad alfa de Cronbach con los datos del presente estudio fue de .85 para la escala total de 14 reactivos.

El segundo instrumento fue el Cuestionario sobre Estilos de Mensajes en el Manejo de Conflictos (Ross-DeWine Conflict Management Message Style Instrument, CMMS; Ross y De Wine, 1988). Se utilizó la versión castellana validada por Luna y Laca (2014), la cual tiene 13 reactivos divididos en tres escalas: Estilo centrado en uno mismo (tres reactivos), Estilo centrado en la otra parte (cuatro reactivos), y Estilo centrado en el problema (seis reactivos). El formato de respuesta es tipo Likert de cinco puntos, desde 1 = Nunca hasta 5 = Siempre. Se calcula la media aritmética de las respuestas de los participantes en cada escala. La confiabilidad alfa de Cronbach con los datos del presente estudio fue de .74, .71 y .83 para las mencionadas tres escalas, respectivamente.

\section{Resultados}

En la Tabla 1 se presentan los puntajes obtenidos por los participantes en las variables de estudio por grupos de género, edad y carrera de estudio, así como para la muestra total. Como se puede observar, las puntuaciones del estilo enfocado en el problema presentan una tendencia a ubicarse por encima del valor central de la escala del cuestionario CMMS (3 = algunas veces), mientras que las del Estilo enfocado en la otra parte se ubican por encima del segundo nivel ( 2 = rara vez), y las del Estilo enfocado en sí mismo están más próximas al nivel 1 de dicha escala ( 1 = ninguna vez). Por su parte, el puntaje de Atención plena disposicional se ubicó en un punto intermedio entre el nivel 2 y el 3 de su correspondiente escala (experimentar situaciones de atención plena ocasionalmente y con frecuencia, respectivamente). 
Tabla No. 1: Medias y desviaciones estándar de los puntajes de los participantes en las variables de estudio, según género, edad y carrera

\begin{tabular}{ccccc}
\hline & $\begin{array}{c}\text { Atención plena } \\
\text { disposicional } \\
\text { M (DE) }\end{array}$ & $\begin{array}{c}\text { Enfocado en sí } \\
\text { mismo } \\
\text { M (DE) }\end{array}$ & $\begin{array}{c}\text { Enfocado en la } \\
\text { otra parte } \\
\text { M (DE) }\end{array}$ & $\begin{array}{c}\text { Enfocado en el } \\
\text { problema } \\
\text { M (DE) }\end{array}$ \\
\hline $\begin{array}{c}\text { Hombres } \\
\text { Mujeres }\end{array}$ & $2.88(0.42)$ & $1.45(0.68)$ & $2.76(0.82)$ & $3.44(0.81)$ \\
\hline 17 a 19 años & $2.72(0.53)$ & $1.42(0.67)$ & $2.67(0.73)$ & $3.61(0.87)$ \\
20 y 21 años & $2.85(0.50)$ & $1.49(0.66)$ & $2.67(0.90)$ & $3.66(0.79)$ \\
22 a 25 años & $2.71(0.55)$ & $1.47(0.74)$ & $2.51(0.91)$ & $3.59(0.82)$ \\
\hline Total & $2.77(0.53)$ & $1.46(0.68)$ & $2.62(0.85)$ & $3.57(0.86)$ \\
\hline
\end{tabular}

Fuente: elaboración propia, resultado de investigación.

En el análisis correlacional de Pearson, la varible de Atención plena disposicional presentó correlaciones estadísticamente significativas con Estilo enfocado en sí mismo $\left(r{ }_{(298)}=-.13, p<.05\right)$ y Estilo enfocado en el problema $\left(r_{(300)}=.41, p<.01\right)$. La correlación con el Estilo enfocado en la otra parte resultó débil y no significativa ( $r$ $\left.{ }_{(300)}=.04, p<.50\right)$.

\section{Discusión}

Con relación al primer objetivo específico de la presente investigación, tal como se muestra en los resultados, se percibe una cierta inclinación hacia puntajes moderados de atención plena disposicional y manejo de conflictos cooperativo o enfocado en el problema, ya que los promedios de dichas variables tendieron a ubicarse por encima de los valores centrales de las correspondientes escalas de respuesta. Este resultado es congruente con algunos estudios en los que se ha medido la atención plena disposicional en población joven y adulta en México utilizando el mismo instrumento empleado en el presente estudio (Laca et al., 2017; Pérez-Verduzco y Laca Arocena, 2017), así como con los resultados de otros estudios realizados con jóvenes en que se ha utilizado el cuestionario 
CMMS (Mejía y Laca, 2006; Laca y Mejía, 2017; Luna y Laca, 2014). Estos resultados indican, por un lado, un desarrollo espontáneo de las capacidades de manejo cooperativo de conflictos y de atención plena, aunque de grado moderado, en las interacciones de la vida ordinaria de los jóvenes. Por otro lado, el grado moderado de este desarrollo sugiere la conveniencia de implementar intervenciones sistemáticas dirigidas a fortalecer las capacidades mecionadas, a fin de promover un mayor fortalecimiento de las mismas.

Con relación al segundo objetivo, relativo al análisis correlacional, de acuerdo con el conocido criterio de Cohen (1988), una correlación de .1 se estima débil, una de .3 se valora como moderada y una de .5 o más como fuerte. Por lo cual, se puede afirmar que, en el presente estudio, la atención plena disposicional presentó una correlación positiva directa estadísticamente significativa, de moderada a fuerte, con el estilo cooperativo, así como una correlación significativa inversa, aunque débil, con el estilo dominante. Estos resultados coinciden con los de Laca y Mejía (2017) y Pérez-Yus et al. (2020), en cuanto a documentar en personas jóvenes y adultas una relación positiva entre manejo cooperativo de conflictos y atención plena. En ese marco, el presente estudio aporta nueva información sobre dicha relación, ampliando el conocimiento que actualmente se tiene de la misma hacia el contexto de las interacciones entre jóvenes universitarios en el espacio escolar.

En conclusión, como se pudo observar, la presente investigación contribuye a extender el estado del conocimiento que se posee en la actualidad acerca de las relaciones entre los estilos de manejo de conflictos interpersonales y la atención plena disposicional, al estudiar dicha vinculación en el ámbito de las relaciones entre pares estudiantes de pregrado. Por tanto, este trabajo constituye una aportación de interés para futuros programas de investigación e intervención que pudieran desarrollarse para el trabajo con jóvenes, especialmente en el marco de la enseñanza universitaria.

Dentro de las limitaciones del presente estudio cabe mencionar que estuvo circunscrito únicamente a las relaciones entre jóvenes universitarios compañeros de escuela, por lo que cabe para futuros trabajos considerar otros miembros de la comunidad 
universitaria, así como ampliar la mirada hacia la comprensión de las dinámicas comunitarias y sociales que pudieran están también influyendo en el desarrollo de las competencias para el manejo constructivo de conflictos.

Finalmente, debe destacarse que la atención plena fue abordada en la presente investigación en su faceta disposicional (esto es, como una capacidad ya dada y producida espontáneamente en los individuos), a diferencia de otros trabajos en los que se ha abordado el estudio del mindfulness a partir de la evaluación de programas de entrenamiento. Por tanto, el presente trabajo contribuye al conocimiento de un aspecto de la atención plena que ha sido escasamente abordado en la investigación, considerando especialmente su relación con los estilos de manejo de conflictos en jóvenes universitarios.

\section{Agradecimientos}

Los investigadores agradecemos la colaboración de los colegas y colaboradores de los Cuerpos Académicos "Adolescentes: mundo y vida" (UDG-CA-967) y "Violencia, manejo del conflicto y mediación" (UDG-CA-705) de la Universidad de Guadalajara, por su apoyo y facilidades brindadas para la realización del presente estudio, así como a los estudiantes Sergio Alejandro Cervantes Ramírez, María Isabel Chacón Cruz, Daniel Martínez Ceja y Mariana Jacquelin Solares Romero, de la Licenciatura en Psicología, su valiosa ayuda en la captura de los cuestionarios. 


\section{Referencias}

Cohen, J. (1988). Statistical power analysis for the behavioral sciences (2nd. ed.). Lawrence Erlbaum Associates. http:// www.utstat.toronto.edu/ brunner/oldclass/378f16/ readings/CohenPower.pdf

Laca, F. A. y Mejía, J. C. (2017). Dependencia emocional, consciencia del presente y estilos de comunicación en situaciones de conflicto con la pareja. Enseñanza e Investigación en Psicología, 22(1), 66-75. https://www. redalyc.org/pdf/292/29251161006.pdf

Laca, F. A., Mejía, J. C., Rodríguez, E., y Carrillo, E. (2017). Avances en psicología del bienestar subjetivo: Relación entre el mindfulness y la satisfacción con la vida. Uaricha. Revista de Psicología, 14(32), 78-86. http://www.revistauaricha. umich.mx/index.php/urp/article/view/154/141

Levesque, R. J. R. (2018). Mindfulness meditation. In R. J. R. Levesque (Ed.), Encyclopedia of adolescence (2a. ed.; pp. 2349-2355). Springer. https://doi.org/10.1007/9783-319-33228-4_445

López-Hernáez, L. (2016). Técnicas mindfulness en centros educativos. Desarrollo académico y personal de sus participantes. Revista Española de Orientación y Psicopedagogía, 27(1), 134-146. https://doi.org/10.5944/ reop.vol.27.num.1.2016.17033

Luna, A. C. A. y Laca, F. A. (2014). Estilos de mensajes en el manejo de conflictos en adolescentes y jóvenes mexicanos. Boletín de Psicología, 110, 37-51. https:// www.uv.es/seoane/boletin/previos/N110-3.pdf

Mejía, J. C. y Laca, F. A. (2006). Estilos de comunicación en el conflicto y confianza en las propias decisiones. Enseñanza e Investigación en Psicología, 11(2), 347-358. https:// www.redalyc.org/pdf/292/29211210.pdf 
Pérez-Verduzco, G. y Laca-Arocena, F. A. (2017). Traducción y validación de la versión abreviada del Freiburg Mindfulness Inventory (FMI-14). Revista Evaluar, 17(1), 8093. https://doi.org/10.35670/1667-4545.v17.n1.17076

Pérez-Yus, M. C., Ayllón-Negrillo, E., Delsignore, G., MagallónBotaya, R., Aguilar-Latorre, A., y Oliván Blázquez, B. (2020). Variables associated with negotiation effectiveness: The role of mindfulness. Frontiers in psychology, 11, 1214. https://doi.org/10.3389/fpsyg.2020.01214

Ramírez-Garduño, A., Veytia, M., Guadarrama, R., y Lira, J. (2020). Mindfulness disposicional, espiritualidad y religión y su papel como factores protectores del consumo de sustancias en jóvenes mexicanos. Nova Scientia. Revista de Investigación de la Universidad De La Salle Bajío, 12(2), 1-23. https://doi.org/10.21640/ns.v12i25.2460

Ross, R. G. y DeWine, S. (1988). Assessing the Ross-DeWine Conflict Management Message Style (CMMS). Management Communication Quarterly, 1(3), 389-413. https://doi.org/10.1177/0893318988001003007

Soriano, J. G., Pérez-Fuentes, M. C., Molero-Jurado, M. M., Gázquez, J. J., Tortosa, B. M., y González, A. (2020). Beneficios de las intervenciones basadas en la atención plena para el tratamiento de síntomas ansiosos en niños y jóvenes: Metaanálisis. Revista Iberoamericana de Psicología y Salud, 11(1), 42-53. https://doi. org/10.23923/j.rips.2020.01.034

Toniolo-Barrios, M., Brasil, A. y Pitt, L.F. (2020). Nine Prolific Years: An Analysis of Publications in Mindfulness. Mindfulness, 11, 1077-1089. https://doi.org/10.1007/s12671-02001321-w

Walach, H., Buchheld, N., Buttenmüller, V., Kleinknecht, N., y Schmidt. S. (2006). Measuring mindfulness—the Freiburg Mindfulness Inventory (FMI). Personality and Individual Differences, 40(8), 1543-1555. https://doi.org/10.1016/j. paid.2005.11.025 


\section{Sobre los autores}

\section{Alejandro César Antonio Luna Bernal}

Es Doctor en Psicología por la Universidad de Colima, México. Profesor Titular de Tiempo Completo adscrito al Departamento de Filosofía de la Universidad de Guadalajara (México). Miembro del Sistema Nacional de Investigadores (SNI, Nivel I) del Consejo Nacional de Ciencia y Tecnología (CONACYT). Integrante del Cuerpo Académico Adolescentes: mundo y vida (UDG-CA-967).

\section{Josefina Sandoval Martínez}

Es Doctora en Psicología por la Universidad de Guadalajara. Profesora Titular de Tiempo Completo adscrita al Departamento de Psicología Aplicada de la Universidad de Guadalajara (México). Integrante del Cuerpo Académico de Violencia, Manejo de Conflicto y Mediación (UDG-CA-705) y Coordinadora de Planeación del Departamento de Psicología Aplicada.

\section{Alejandra De Gante Casas}

Es Doctoranda en Psicología por la Universidad de Guadalajara, con Maestría en Salud Pública. Profesora-investigadora adscrita al Departamento de Psicología Aplicada de la Universidad de Guadalajara. Integrante del Cuerpo Académico de Violencia, Manejo de Conflicto y Mediación (UDG-CA-705). 